\title{
Valorization of platelets with no therapeutic value with Pulsed Electric Fields *
}

\author{
D Rego, LM Redondo, AP Sousa, S Abreu, M Serra, VE Santo
}

\begin{abstract}
Platelets are blood components with high biomedical potential due to their physiological role in wound healing and their rich growth factor content. This paper describes proof of concept experiments aimed to produce a new blood derived product by applying Pulsed Electric Fields (PEF) in platelet concentrates with no therapeutic value for transfusion medicine. A human platelet concentrate suspension was subjected to a PEF treatment of 1 pulse, $5 \mathrm{kV} / \mathrm{cm}$, with $2 \mu \mathrm{s}$. Release of Platelet Derived Growth Factor (PDGF) from the electroporated platelets was measured by ELISA. Furthermore, the biological activity of the obtained blood derived product was characterized. Human mesenchymal stem cells (hMSC) were cultured in the presence of the proteins released from the platelets after PEF application and evaluated for their expansion potential.

Results show that platelets concentrates submitted to a single PEF treatment can release PDGF to the supernatant. Protein release from the single and transient $P E F$ cycle was confirmed by the expansion of hMSC cultured with a medium supplemented with platelet releasate obtained from electroporated platelets. These results support the valorization of platelet concentrates with no transfusion value that would otherwise be discarded. Applying PEF might allow the development of alternative releasate for different biomedical applications, namely stem cells culture.
\end{abstract}

\section{INTRODUCTION}

Platelets constitute a vital component of blood, primarily responsible for bleeding cessation. Standard blood bank procedures are designed to keep Platelet Concentrates (PC) viable and functional by strict room temperature storage requirements and continuous agitation. Nevertheless, PC only have a 5-day shelf-life (or 7-day if submitted to pathogen reduction), and when stored at $20-24^{\circ} \mathrm{C}$ with continuous agitation [26, 27]. Expired platelets are no longer suitable for transfusion, but they still have the potential for alternative biomedical applications. Converting these PC, with no transfusional value, into new biological blood-derivative product with high biomedical potential is one possibility for blood banks diversification in terms of Cellular Therapies and Regenerative Medicine.

Platelets are also known for their enriched content in bioactive factors. Fundamental in vitro studies have been carried out over the years to understand the mechanisms of growth-promoting and tissue-healing capacity of plateletderived factors $[1,2,3]$. When activated in our body, platelets release a group of bioactive molecules that promote the recruitment, growth, and morphogenesis of cells, wound

\footnotetext{
*Research supported by FCT PTDC/BTM-ORG/32187/2017. D. Rego is with EnergyPulse Systems, Lisbon, Portugal (e-mail: duarte.rego@energypulsesytems.com).

LM Redondo, is with ISEL, Lisbon, Portugal (e-mail: lmredondo@deea.isel.ipl.pt).
}

healing and tissue regeneration [4-5]. Some of the factors found inside the platelets comprise isoforms of PDGF, Insulin Growth Factor (IGF), Transforming Growth Factor (TGF), known to contribute for stem cell expansion and differentiation into different lineages such as osteoblasts [6, 7]. In fact, PC can be used as an alternative source of growth factors for therapeutic applications and constitute a very exciting area of research. In the past two decades, the increasing knowledge on the physiological role of platelets in wound healing and tissue injury suggests the potential of using activated platelets as topical therapeutic tool [8].

Considerable effort has been done to evaluate the role of Platelet Lysate (PL), obtained after activation of platelets and consequent release of their intracellular protein content, as a supplement for in vitro cell culture. PL has shown to accelerate cell expansion in vitro, thus reducing the duration of ex vivo manipulation [9-11] and proving its capacity to replace fetal bovine serum (FBS), the conventional animal source supplement.

Platelet activation can be initiated by several methods, such as shear forces, or contact with collagen and thrombin [12]. Calcium also activates platelets as it replaces the calcium bound by the anticoagulant agents, used to avoid coagulation of the collected whole blood [13]. Exogenous thrombin can also be provided; nevertheless the use of animal-derived thrombin has raised concerns regarding safety of the final product [1]. Studies also show that using thrombin fails to maximize the potential of PL [2]. Freeze and thaw cycles are also used for activation of these blood components. The rate of activation is quite successful as these temperature cycles induce lysis of the cells [6]. However, this method leads to irreversible activation of platelets, with poor control over the final obtained product. Therefore, other activation stimuli are required to overcome these limitations.

The application of pulsed electric fields (PEF) in biological cell systems is a known technique to induce cell membrane permeabilization, called electroporation [14-20]. This technology is already being proposed to enable endogenous proteins to be released in platelets [21-24]; however no studies have demonstrated the ability to define a specific pulse to achieve certain final protein content.

In this study, a proof-of-concept for the valorization of PC with no transfusion value is presented. The goal is to propose a new methodology for producing a new blood-derived product for non-transfusion applications, with high therapeutic

AP Sousa is with IPST, Lisbon, Portugal.

$\mathrm{S}$. Abreu is with ITQB, Oeiras, Portugal.

M Serra is with iBET/ITQB, Oeiras, Portugal.

VE Santo was with iBET/ITQB, Oeiras, Portugal and is now with JUST, San Francisco, CA, USA. 
and commercial value, where the high potential of PEF technology is expected to contribute for this methodology. In this first study, a platelet concentrate with no transfusion value was subjected to a PEF treatment consisting of one $5 \mathrm{kV} / \mathrm{cm}$ pulse with $2 \mu \mathrm{s}$ and the release of PDGF was measured by ELISA. Furthermore, the biological activity of the obtained platelet releasate was characterized. hMSC were cultured in the presence of medium supplemented with platelet releasate after electroporation with $\mathrm{PEF}$ and evaluated for their expansion and differentiation potential.

\section{METHODS}

\section{A. Human Platelet Concentrates (PC)}

PC were made available by the Portuguese Institute for Blood and Transplantation (IPST, Lisbon, Portugal) from material not suitable for transfusion. PC follow a well-defined composition of more than $2 \times 1011$ platelets per unit (11350 $\mathrm{mL}$ ); less than 1 x 106 white blood cells and less than $3 \times 109$ red blood cells. For this study, PC were kept in agitation and $20-24^{\circ} \mathrm{C}$ between 6 and 10 days.

\section{B. Freeze \&Thaw cycles}

Part of the collected samples were subjected to three repeated temperature cycles: samples were frozen with liquid nitrogen at $-196{ }^{\circ} \mathrm{C}$ and thawed at $37{ }^{\circ} \mathrm{C}$ in a sterile water bath). After the temperature cycles, samples were stored at $-20{ }^{\circ} \mathrm{C}$ until further use. Before storing at $-20{ }^{\circ} \mathrm{C}$, samples were centrifuged (1400 xg, $10 \mathrm{~min})$ to remove remaining platelets and platelet debris. These samples were called PL F\&T (PC lysate by freeze $\&$ thaw cycles).

\section{PEF application}

EPULSUS $\AA-L P M 1-10$ (EnergyPulse Systems, Lisbon, Portugal) PEF generator was used to apply the PEF protocol. Pulse voltage and current were recorded with a Tektronix TPS2024B 200MHz 2GS/s oscilloscope, using PMK PHV1000 100:1 400MHz 50M $\Omega / / 7.5 \mathrm{pF}$ high voltage probe and current transformer model 0.5-0.01 (Stangenes Industries Inc, Palo Alto, CA). PEF treatments were applied to a parallel plate titanium electrodes treatment chamber with $1 \mathrm{~cm}$ gap and a surface area of $1 \mathrm{~cm} 2$. Batches of $1 \mathrm{~mL}$ were treated with 1 pulse of $2 \mu \mathrm{s}$ at $5 \mathrm{kV} / \mathrm{cm}$. Other protocols, with higher electric fields, were tested unsuccessfully due to breakdown problems on the surface of the treated volume, due to the design of the treatment chamber.

After PEF application, PC was removed from treatment chamber and centrifuged in $1.5 \mathrm{~mL}$ Eppendorf tube $(1400 \mathrm{xg}$, $10 \mathrm{~min}$ ) to remove debris and remaining platelets. . Resulting supernatant (releasate) was stored at $-20{ }^{\circ} \mathrm{C}$ until further analysis. These samples were called PR PEF (PC releasate treated with PEF).

PC samples were also processed without PEF (PR wo act). These samples were used to isolate the effect of PEF from the rest of the process (centrifugation, age of culture, others).

\section{Growth Factor Quantification}

PDGF release was measured using ELISA kits (Human PDGF-BB Quantikine ELISA Kit, bio-techne/R\&D Systems, Minneapolis, MN, USA) to determine the concentration of PDGF in the samples. The protocol was followed according to kit instructions. Optical density was read at $450 \mathrm{~nm}(\mathrm{n}=3)$ using a multi-well plate reader (Tecan).

\section{A. hMCS Culture}

The releasate obtained from PEF exposure was tested for biocompatibility and bioactivity using hMSC isolated from the bone marrow (donor 00055; RoosterBio, Maryland, USA).

Five different conditions were used to compare the bioactivity of the PEF releasate:

a. Control 1 - cells cultured in MesencultTM-XF on a surface coated with MesencultTM- Attachment substrate (both from STEMCELLTMTechnologies, Vancouver, Canada) - optimized medium formulation for hMSC expansion;

b. Control 2 - cells cultured in DMEM-LG $+10 \%$ FBS (ThermoFisherTMScientific, Massachusetts, USA), - animal source, commercially alternative to human platelets derivatives;

c. PR wo act - cells cultured in DMEM-LG $+10 \%$ PR without activation;

d. PL F\&T - cells cultured in DMEM-LG $+10 \% \mathrm{PL}$ submitted to freeze/thaw cycles ;

e. PR PEF - cells cultured in DMEM-LG $+10 \%$ PR treated by PEF.

In all conditions, the medium was supplemented with 2 $\mathrm{mM}$ of glutamine (Life Technologies, USA).

hMSCs were inoculated at a density of $4500 \mathrm{cell} / \mathrm{cm} 2$ in adherent static culture systems (12- and 48- well plates), and cultured for 7 days at $37^{\circ} \mathrm{C}$ in a humidified atmosphere of $5 \%$ $\mathrm{CO} 2$ and $95 \%$ air.

Medium was changed at day 4. Cultures were monitored in terms of:

a. Cell adhesion efficiency $-24 \mathrm{~h}$ after inoculation; by counting the cells in a hemocytometer using trypan blue exclusion test. Cell adhesion efficiency was estimated by calculating the ratio between the number of cells attained $24 \mathrm{~h}$ after inoculation with the initial cell number at the inoculum.

b. Cell growth - cell concentration was determined at day 1,4 and 7 by counting the cells in a hemocytometer using trypan blue exclusion test. Cell expansion factor was estimated by calculating the ratio between the number of cells attained at day 7 with the cell number estimated at day 1 ;

c. Cell morphology - day 1, day 4 and day 7; by phase contrast microscopy;

Methods used for cell culture and characterization were performed according to previous publication [25]. All tests were performed in duplicates.

\section{RESULTS AND DISCUSSION}

\section{A. PDGF quantification}

The release of PDGF from the platelets was quantified by ELISA and results are presented in Fig 1. PR wo act show the lowest value $(8.59 \pm 0.05 \mu \mathrm{g} / \mathrm{mL})$ followed by PR PEF with $9.08 \pm 0.10 \mu \mathrm{g} / \mathrm{mL}$ and PL F\&T with $12.78 \pm 0.32 \mu \mathrm{g} / \mathrm{mL}$. Against PR wo act, PR PEF has an increase of $6 \%$ on PDGF concentration and PL F\&T has an increase of $48 \%$ on PDGF concentration. Although, is only a small difference between PR wo act and PR PEF, standard deviation of the analyses points to a significant difference between the two values and 
were interpreted here as a difference promoted by the exposure to PEF. However, against PDGF value obtained with F\&T treatment (PL F\&T), one conclude that the release (PR PEF) is only partial, since the values are still considerably lower. The difference in PDGF concentration measured suggests that only part of the platelets population is being affected by the PEF treatment. Another hypothesis is that PEF can only promote a partial release of the components. Only with the available results of this work it is not possible to conclude on this subject and further optimization of the PEF protocol is necessary.

Nevertheless, the tested PEF protocol shows it is possible

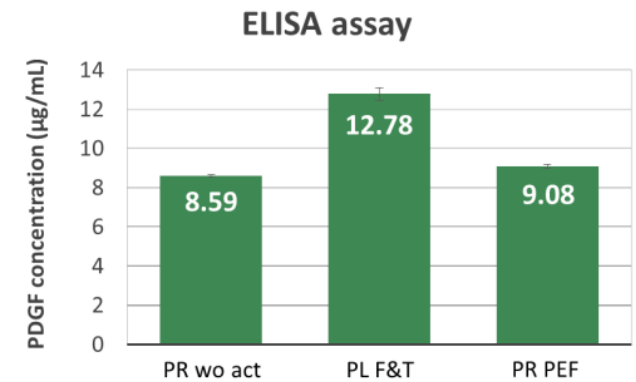

Figure 2. PDGF concentration $(\mu \mathrm{g} / \mathrm{mL})$ for different processing conditions.

to promote release of components using only pulsed electric fields as an alternative to thrombin or $\mathrm{Ca} 2+$ described by [22]. We also believe that this proof-of-concept study shows that there is potential to develop more elegant releasate methods that trigger selective release of platelets without inducing complete disruption of cell membrane.

B. hMSC culture

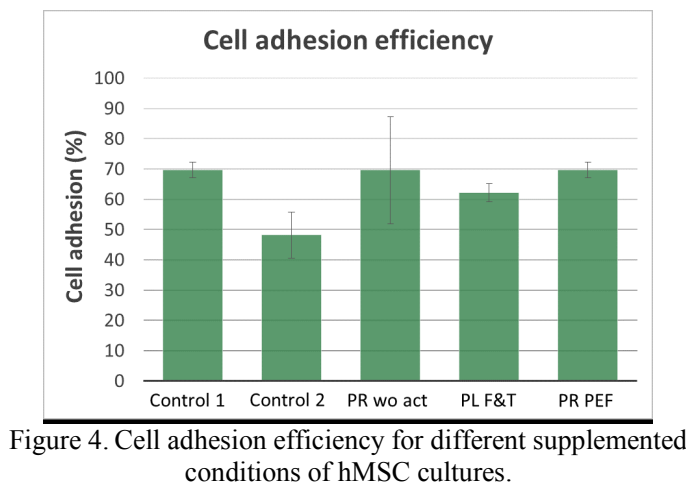

Cell adhesion efficiency - After the first day of culture, it is possible to see that cell adhesion efficiency was high in all cultures supplemented with PL and PR (Fig. 2). The values obtained for the three conditions were very similar between them and to cells cultured in MesencultTM-XF (Control 1). This observation demonstrates that, in general, growth factors and other molecules kept their biological activity after PEF treatment, because (PR PEF)-supplemented cultures showed proportional growth in relation to PL F\&T and PR wo act. This observation is important as a start point to understand that electric field applied is not changing the structure of the proteins (such as PDGF and other growth factors). The lowest percentage of adherent cells was attained for cultures with DMEM-LG supplemented with 10\% FBS (Control 2).

Cell growth - From Fig. 3 and Fig. 4 it is possible to observe that cell proliferation was higher in all PL and PR supplemented medium conditions than in FBS supplemented medium. Furthermore, both PL F\&T and PR PEF samples show higher concentration levels than cells supplemented with PR wo act. This supports the idea that the composition of PR PEF is more similar to PL F\&T than PR wo act and that this composition is beneficial for hMSC growth. This observation is also in accordance with the PDGF quantification results, where a small increase of $6 \%$ on the PDGF concentration expresses a small increase on the cell growth on the hMSC cultures. Only the cells that were cultured with PL F\&T showed an expansion factor similar to Control 1. These results supports the idea that it is possible to present a natural, human origin, culture medium with a performance similar to the synthetic optimized MesencultTM-XF, needed for biomedical applications.

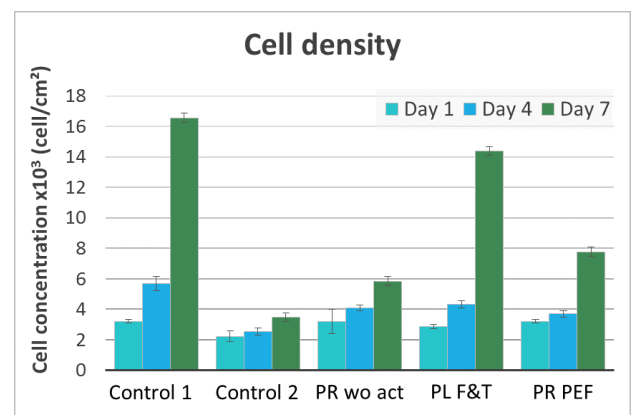

Figure 1. Cell density (cell/cm2) for all tested conditions.

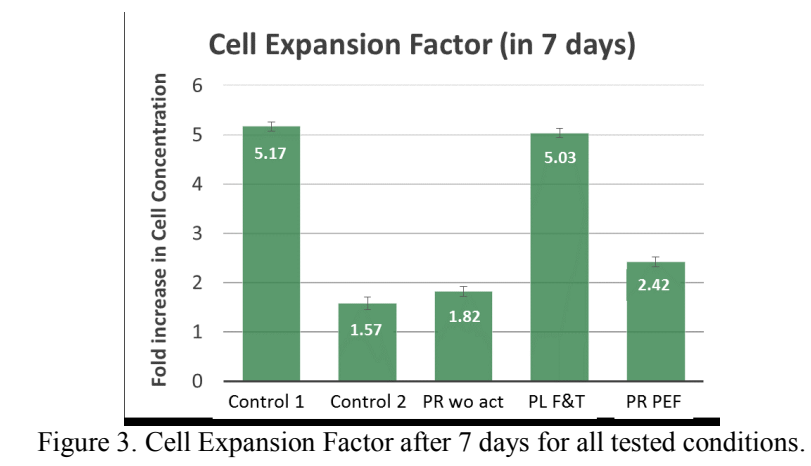

Cell Morphology - hMSC show different morphologies in the different culture conditions evaluated. In particular, cells display a star-shaped with accentuated membrane regions when cultured in PL and PR supplemented media. In Control 1 cells showed an elongated, fibroblastic-like, spindle-shaped morphology whereas in Control 2 some flattened and star-like shape cells with prominent nucleus were observed. This observation supports the importance of creating products that are human derived, not only for ethical reasons but also to improve the quality of all products produced downstream. 


\section{CONCLUSION}

In conclusion, this work demonstrates the potential of $\mathrm{PEF}$ for the transformation of a PC with no transfusional application into a new and valuable product for biomedical applications (platelet releasate). However, further work must be done, in order to optimize PEF application and making it a real alternative technology for industrial scale production of such products. Next steps should focus on optimizing the PEF protocol and treatment chamber design in order to increase the release of intracellular components from the platelets.

PEF protocol should also be used in an attempt to selectively activate platelets to extract specific molecules. For this, more studies should be done focusing on specific growth factors or other molecules of interest for biomedical and biotechnology applications.

\section{ACKNOWLEDGMENT}

Authors like to thank to Fundação para a Ciência e a Tecnologia project grant PTDC/BTM-ORG/32187/2017.

\section{REFERENCES}

[1] Nair M.B. Varma H.K. John A. Platelet-rich plasma and fibrin gluecoated bioactive ceramics enhance growth and differentiation of goat bone marrow-derived stem cells. Tissue Eng Part A. 2009;15:1619.

[2] Sell S.A. Wolfe P.S. Ericksen J.J. Simpson D.G. Bowlin G.L. Incorporating platelet-rich plasma into electrospun scaffolds for tissue engineering applications. Tissue Eng Part A. 2011;17:2723.

[3] Wirz, Simone, et al. "Influence of platelet-derived growth factor-AB on tissue development in autologous platelet-rich plasma gels." Tissue Engineering Part A 17.13-14 (2011): 1891-1899.

[4] Laura Mazzucco et al." Platelet-Derived factors involved in Tissue Repair-From signal to function”, Transfusion Medicine Reviews, 24, 3, 2010:218-234.

[5] Anitua E. Sanchez M. Orive G. Potential of endogenous regenerative technology for in situ regenerative medicine. Adv Drug Deliv Rev. 2010;62:74.

[6] Santo VE, Gomes ME, Mano JF, Reis RL. Chitosan/Chondroitin sulphate nanoparticles for controlled delivery of platelet lysates in bone regenerative medicine. Journal of Tissue Engineering and Regenerative Medicine, 2012, 6 (Suppl 3) s47-59.

[7] Santo VE, Duarte ARC, Popa EG, Gomes ME, Mano JF, Reis RL. Enhancement of osteogenic differentiation of human adipose derived stem cells by the controlled release of platelet lysate from hybrid scaffolds produced by supercritical fluid foaming. Journal of Controlled Release, 2012, 162(1) 19-27.

[8] Anitua E. Sanchez M. Nurden A.T. Nurden P. Orive G. Andia I. New insights into and novel applications for platelet-rich fibrin therapies. Trends Biotechnol. 2006;24:227.

[9] Zaky S.H. Ottonello A. Strada P. Cancedda R. Mastrogiacomo M. Platelet lysate favours in vitro expansion of human bone marrow stromal cells for bone and cartilage engineering. J Tissue Eng Regen Med. 2008;2:472.

[10] Salvadè A. Mina P.D. Gaddi D. Gatto F. Villa A. Bigoni M., et al. Characterization of platelet lysate cultured mesenchymal stromal cells and their potential use in tissue-engineered osteogenic devices for the treatment of bone defects. Tissue Eng Part C Methods. 2009;16:201.

[11] Santo VE, Popa EG, Gomes ME, Mano JF, Reis RL. Natural assembly of platelet lysate-loaded nanocarriers into enriched 3D hydrogels for cartilage regeneration. Acta Biomaterialia, 2015, 19, 56-65.

[12] Blair P. Flaumenhaft R. Platelet alpha-granules: basic biology and clinical correlates. Blood Rev. 2009;23:177.

[13] Intini G. The use of platelet-rich plasma in bone reconstruction therapy. Biomaterials. 2009;30:4956.

[14] Weaver JC: Electroporation of biological membranes from multicellular to nano scales. ITDEI. 2003, 10: 754-768. 10.1109/TDEI.2003.1237325.
[15] Weaver JC: Electroporation: a general phenomenon for manipulating cells and tissues. J Cell Biochem. 1993, 51: 426-435.

[16] Gothelf A, Mir LM, Gehl J: Electrochemotherapy: results of cancer treatment using enhanced delivery of bleomycin by electroporation. Cancer Treat Rev. 2003, 29: 371-387. 10.1016/S0305-7372(03)000732.

[17] Davalos RV, Mir IL, Rubinsky B: Tissue ablation with irreversible electroporation. Ann Biomed Eng. 2005, 33: 223-231. 10.1007/s10439005-8981-8.

[18] Edd JF, Horowitz L, Davalos RV, Mir LM, Rubinsky B: In vivo results of a new focal tissue ablation technique: irreversible electroporation. IEEE Trans Biomed Eng. 2006, 53: 1409-1415. 10.1109/TBME.2006.873745.

[19] Rubinsky B: Irreversible electroporation in medicine. Technol Cancer Res Treat. 2007, 6: 255-260.

[20] Schoenbach KH, Hargrave B, Joshi RP, Kolb JF, Nuccitelli R, Osgood C, Pakhomov A, Stacey M, Swanson RJ, White JA, et al: Bioelectric effects of intense nanosecond pulses. ITDEI. 2007, 14: 1088-1109. 10.1109/TDEI.2007.4339468.

[21] Allen L. Garner et al. "Ex vivo platelet activation with extended duration pulse electric fields for autologous platelet gel applications", EWMA Journal 2015 vol 15 no 1., 15-19.

[22] A. P Sousa, Marcos T Pereira, Luis M Redondo. "Impact of Pulsed Electric Fields in Standard Platelet Components", 11th International Bioelectrics Symposium, Columbia, 2014.

[23] Jue Zhang et al. "Nanosecond pulse electric field (nanopulse): A novel non-ligand agonist for platelet activation, Archives of Biochemistry and Biophysics 471 (2008) 240-248.

[24] Andrew S Torres et al. "Platelet activation using electric pulse stimulation: growth factor profile and clinical implications", J Trauma Acute Care Surg., 77, 3, Supplement 2.

[25] Cunha B, Aguiar T, Carvalho SB, Silva MM, Gomes RA, Carrondo MJT, Gomes-Alves P, Peixoto C, Serra M, Alves PM. (2017) "Bioprocess integration for human mesenchymal stem cells: From up to downstream processing scale-up to cell proteome characterization", Journal of Biotechnology 20 (248):87-98.

[26] Direção Geral da Saúde, "Norma no 010/2012 de 16/12/2012: Utilização Clínica de Concentrados Plaquetários no Adulto", (2012), 3.

[27] European Directorate for the Quality of Medicines \& HealthCare (EDQM), "The Guide to the preparation, use and quality assurance of blood components", 19th edition (2017). 